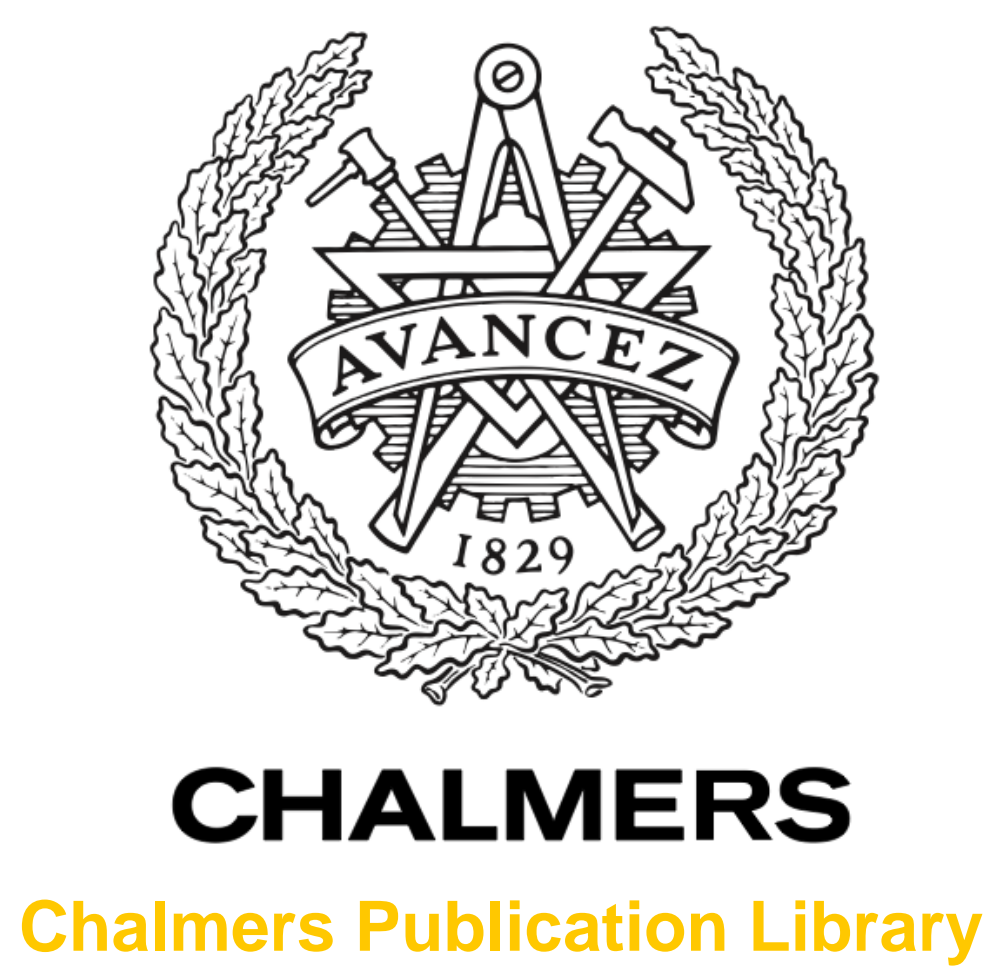

\title{
Power Allocation for Two-Cell Two-User Joint Transmission
}

This document has been downloaded from Chalmers Publication Library (CPL). It is the author's version of a work that was accepted for publication in:

IEEE Communications Letters (ISSN: 1089-7798)

Citation for the published paper:

Li, J. ; Eriksson, T. ; Svensson, T. (2012) "Power Allocation for Two-Cell Two-User Joint

Transmission". IEEE Communications Letters, vol. 16(19), pp. 1474-1477.

$\frac{\text { http://dx.doi.org/10.1109/LCOMM.2012.072012.12 }}{1096}$

Downloaded from: http://publications.lib.chalmers.se/publication/161407

Notice: Changes introduced as a result of publishing processes such as copy-editing and formatting may not be reflected in this document. For a definitive version of this work, please refer to the published source. Please note that access to the published version might require a subscription.

Chalmers Publication Library (CPL) offers the possibility of retrieving research publications produced at Chalmers University of Technology. It covers all types of publications: articles, dissertations, licentiate theses, masters theses, conference papers, reports etc. Since 2006 it is the official tool for Chalmers official publication statistics. To ensure that Chalmers research results are disseminated as widely as possible, an Open Access Policy has been adopted.

The CPL service is administrated and maintained by Chalmers Library. 


\title{
Power Allocation for Two-Cell Two-User Joint Transmission
}

\author{
Jingya Li, Thomas Eriksson, Tommy Svensson and Carmen Botella
}

\begin{abstract}
In this paper, we develop a power allocation scheme for the downlink of a two-cell two-user joint transmission system. The objective is to maximize the sum rate under per-cell power constraints. We study a worst case scenario where the carrier phases between the two base stations are un-synchronized, so that joint transmission must be performed without precoding. The derived power allocation scheme is remarkably simple, i.e., each cell transmits with full power to only one user. Note that joint transmission is still possible, when two cells select the same user for data transmission. Moreover, we prove that, in this scenario, the joint transmission case happens with higher probability when the maximum transmit power is high, or the two users are in the overlapped cell-edge area.
\end{abstract}

Index Terms-Power allocation, joint transmission, coordinated multi-point (CoMP), imperfect synchronization

\section{INTRODUCTION}

Transmit power allocation is an effective way for increasing the sum rate of wireless communication systems. It has been proved in [1] that water-filling power allocation is optimal when maximizing the sum rate under a sum power constraint, assuming different data signals are transmitted through orthogonal channels. Considering a broadcast channel that allows different user data to share the same channel, the optimal solution is then achieved by assigning the total power to the only one user with the best channel gain, namely greedy power allocation (GPA) [2]. Due to the neglecting of interference and the sum power constraint, the results in [1], [2] are not readily applicable for a two-cell power allocation analysis. By assuming per-cell power constraints, it has been proved in [3] that the maximum sum rate for a two-cell system is achieved by binary power control (BPC), i.e., each cell either transmits with full power or does not transmit. However, the formulation of the optimization problem in [3] is restricted to the condition that only one user can be served in each cell at a time slot.

Coordinated multi-point (CoMP) joint transmission has been considered as a promising technique to increase the sum rate and cell-edge performance. If both user data and channel state information (CSI) are shared by coordinated base stations (BSs), multiple BSs can jointly provide data transmission to a user and thereby improve the received signal quality. Major setbacks of CoMP joint transmission are, however, the large CSI feedback overhead, the capacity and latency constraints of backhaul links, and the imperfect synchronization between coordinated BSs [4]. A tradeoff between the system performance and the required amount of CSI feedback and backhaul

This work is supported by the EU FP7 project INFSO-ICT-247223 ARTIST4G and the Swedish Agency for Innovation Systems (VINNOVA).

J. Li, T. Ericksson and T. Svensson are with the department of Signals and Systems, Chalmers University of Technology, Gothenburg, Sweden. (email: \{jingya.li, thomase, tommy.svensson\} @ chalmers.se). C. Botella is with the Institute of Robotics and Information \& Communication Technologies (IRTIC), Universitat de València, València, Spain. (email: Carmen.Botella@uv.es). exchange has been pointed out [4], [5]. This tradeoff is one of the reasons for restricting the use of joint transmission to a limited number of cells of the system [6], [7]. In [6], considering a two-cell single user CoMP system, a joint waterfilling power allocation (Jo-WF) is proved to be optimal for maximizing the capacity of a frequency-selective channel. Since different data are assumed to be transmitted through orthogonal channels, the interference does not exist, which simplifies the optimization problem in [6]. Taking interference into account, BPC is proved to be optimal for maximizing the sum rate with joint transmission [7]. However, similar to [3], it is restricted that at most one user is allowed to be served in each cell at a time slot.

In this paper, the power allocation problem is addressed for the downlink of a two-cell two-user joint transmission system, in a scenario where the carrier phases between two BSs are un-synchronized. As we motivate in Section II, in some applications phase noise and frequency drift will lead to that phase synchronization between BSs is very difficult to achieve. For such applications the studied scenario is the most realistic; for other applications, it can be considered as a worst-case scenario. The objective is to maximize the sum rate under per-cell power constraints. The optimal solution for this scenario is shown to be simple, i.e., each cell transmits with full power to only one user. Note that joint transmission is still possible, when two cells select the same user for data transmission. Moreover, we show that dynamic switching between CoMP and Non-CoMP transmission, depending on the channel condition, is the optimal way under a per-cell power-limited condition for the considered scenario.

\section{SySTEM MOdEL}

We consider the downlink of a two-cell two-user CoMP system, where data symbols of the two users are jointly transmitted from the two cells at the same time using the same spectral resource (see Figure 1). In principle, if infinite cooperation between the two cells is enabled, the scenario is effectively a network MIMO broadcast channel, where all communication links (including the interfering ones) are exploited to coherently transmit user data via joint precoding [4]. However, from a practical point of view, a big challenge for the real-world implementation of CoMP joint transmission is the issue of synchronization between coordinated BSs. The carrier phases between coordinated BSs are difficult to be synchronized mainly due to the effect of carrier frequency offset, or/and phase noise from local oscillators in each BS [8], [9]. BSs with un-synchronized oscillators will result in independent time-continuous phase shifts imposed on the links between BSs and UEs. Here, we study a worst case scenario where the phase shift of each link (arising from the oscillator) 


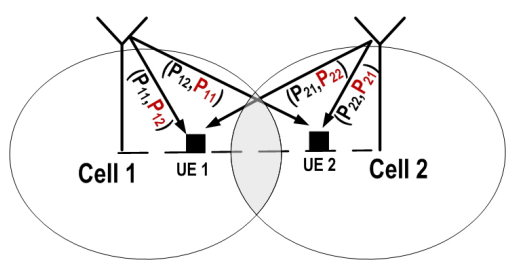

Figure 1. The downlink of a two-cell two-user CoMP system. $(a, b)$ denotes the transmit signal power $a$ and the interference power $b$ on each link.

varies much faster than the channel fading [9], and the phase difference between any pair of links shows a random uniform distribution between $-\pi$ to $\pi$. Note that the performance of joint precoding highly relies on reasonably accurate phase information of the signals that will be received. In the considered scenario, due to the random uniform phasing effect, precoding is not considered when performing joint transmission ${ }^{1}$.

For any given time slot, let $h_{i m}$ denote the channel from cell $i$ to user $m$, which is assumed to be constant over one time slot, with $i=1,2$ and $m=1,2$. Let $\theta_{i m}$ denote the phase shift imposed on $h_{i m}$, which varies over this time slot with $\left(\theta_{i m}-\theta_{j k}\right) \sim U(-\pi, \pi)$, for any $(i, m) \neq(j, k)$. The data symbols, denoted by $x_{1}$ and $x_{2}$, are jointly transmitted from both cells to UE 1 and UE 2 respectively, without precoding. The power allocation across users in cell $i$ is defined by $\mathbf{P}_{i}=\left[P_{i 1}, P_{i 2}\right]$. Each link from cell $i$ to user $m$ contains both the desired data symbols $x_{m}$ with transmit power $P_{i m}$ and the unwanted interference data symbols $x_{k}$ with power $P_{i k}, k \neq m$. Assume that $x_{1}$ and $x_{2}$ are independent Gaussian distributed random variables with zero mean and unit variance. Then, the signal-to-interference-plus-noise ratio (SINR) of user $m$ can be written as ${ }^{2}$

$$
\begin{aligned}
\gamma_{m} & =\frac{E_{\theta, x}\left(\left|\sum_{i=1}^{2} \sqrt{P_{i m}} h_{i m} e^{j \theta_{i m}} x_{m}\right|^{2}\right)}{E_{\theta, x}\left(\left|\sum_{i=1, k \neq m}^{2} \sqrt{P_{i k}} h_{i m} e^{j \theta_{i m}} x_{k}\right|^{2}\right)+\sigma^{2}} \\
& =\frac{\sum_{i=1}^{2} P_{i m} G_{i m}}{\sum_{i=1, k \neq m}^{2} P_{i k} G_{i m}+\sigma^{2}}, m=1,2,
\end{aligned}
$$

where $G_{i m}=\left|h_{i m}\right|^{2}$ and $\sigma^{2}$ is the variance of the independent zero-mean additive white Gaussian noise. By treating interference as noise, the expected sum rate of the two users normalized by the bandwidth can be expressed as

$$
R=\log _{2}\left(\left(1+\gamma_{1}\right)\left(1+\gamma_{2}\right)\right) \text {. }
$$

\section{Optimal Transmit Power Allocation}

The objective is to find an optimal power allocation vector $\mathbf{P}_{i}^{*}$ for each cell $i$ that maximizes the sum rate $R$ subject to per-cell power constraints. Since the logarithm is a monotonically increasing function, the equivalent problem is

$$
\begin{aligned}
\max _{\mathbf{P}_{1}, \mathbf{P}_{2}} J\left(\mathbf{P}_{\mathbf{1}}, \mathbf{P}_{\mathbf{2}}\right) & =\left(1+\frac{\sum_{i=1}^{2} P_{i 1} G_{i 1}}{\sum_{i=1}^{2} P_{i 2} G_{i 1}+\sigma^{2}}\right) \\
& \times\left(1+\frac{\sum_{i=1}^{2} P_{i 2} G_{i 2}}{\sum_{i=1}^{2} P_{i 1} G_{i 2}+\sigma^{2}}\right) \\
\text { s.t. } \Sigma_{m=1}^{2} P_{i m} & \leq P_{i, \max }, P_{i m} \geq 0, i=1,2 .
\end{aligned}
$$

\footnotetext{
${ }^{1}$ The achieved sum rate is a lower bound for a precoded system with perfect synchronization.

${ }^{2}$ Note that only the amplitude information of the channel coefficients are needed, which reduces both feedback and backhaul overhead.
}

Lemma 1. Let $\mathbf{P}_{i}^{*}$ be the optimal transmit power allocation vector, $i=1,2$. Then there is at least one cell index $i$, such that the power constraint for this cell is satisfied with equality.

Proof: Let $\mathcal{F}$ denote the feasible set of (3). Consider a pair of transmit power allocation vectors $\left[\mathbf{P}_{1}, \mathbf{P}_{2}\right] \in \mathcal{F}$ such that for each $i=1,2, \sum_{j=1}^{2} P_{i m}<P_{i, \max }$. Then, it is possible to find a factor $\alpha$, with $\alpha=\min _{i}\left(P_{i, \max } / \sum_{m=1}^{2} P_{i m}\right)>1$, such that at least one of the per-cell power constraints is satisfied with equality, and

$$
\begin{aligned}
J\left(\alpha \mathbf{P}_{1}, \alpha \mathbf{P}_{2}\right) & =\left(1+\frac{\sum_{i=1}^{2} P_{i 1} G_{i 1}}{\sum_{i=1}^{2} P_{i 2} G_{i 1}+\sigma^{2} / \alpha}\right) \\
& \times\left(1+\frac{\sum_{i=1}^{2} P_{i 2} G_{i 2}}{\sum_{i=1}^{2} P_{i 1} G_{i 2}+\sigma^{2} / \alpha}\right) \\
& >J\left(\mathbf{P}_{1}, \mathbf{P}_{2}\right) .
\end{aligned}
$$

Thus, for any power allocation vector $\left[\mathbf{P}_{1}, \mathbf{P}_{2}\right]$ satisfying $\sum_{m=1}^{2} P_{i m}<P_{i, \max }, i=1,2$, it is possible to find a power allocation vector $\left[\alpha \mathbf{P}_{1}, \alpha \mathbf{P}_{2}\right]$ that achieves a larger sum rate, when one of the per-cell power constraints is satisfied with equality, i.e., $\sum_{m=1}^{2} P_{i m}=P_{i, \max }, i=1$ or 2 .

Lemma 2. Let the power constraint for cell $i$ be satisfied with equality, $i=1$ or 2 . Then cell $i$ transmits to only one user with its maximum transmit power.

Proof: Without loss of generality, we assume that the power constraint for cell 1 is satisfied with equality, i.e., $P_{11}+$ $P_{12}=P_{1, \max }$. Thus, for any given feasible transmit power allocation vector $\mathbf{P}_{2}$ of cell 2, the objective function in (3) can be simplified as a function of $P_{11}$

$$
\begin{aligned}
J\left(P_{11}\right) & =\left(\frac{P_{1, \max } G_{11}+\left(P_{21}+P_{22}\right) G_{21}+\sigma^{2}}{\left(P_{1, \max }-P_{11}\right) G_{11}+P_{22} G_{21}+\sigma^{2}}\right) \\
& \times\left(\frac{P_{1, \max } G_{12}+\left(P_{21}+P_{22}\right) G_{22}+\sigma^{2}}{P_{11} G_{12}+P_{21} G_{22}+\sigma^{2}}\right) \\
& =\frac{A}{-P_{11}^{2}+B P_{11}+C},
\end{aligned}
$$

where

$$
\begin{aligned}
A & =\left(P_{1, \max } G_{11}+\left(P_{21}+P_{22}\right) G_{21}+\sigma^{2}\right) \\
& \times\left(P_{1, \max } G_{12}+\left(P_{21}+P_{22}\right) G_{22}+\sigma^{2}\right) / G_{11} G_{12}>0, \\
B & =P_{1, \max }+\left(P_{22} G_{21}+\sigma^{2}\right) / G_{11}-\left(P_{21} G_{22}+\sigma^{2}\right) / G_{12}, \\
C & =\left(P_{1, \max } G_{11}+P_{22} G_{21}+\sigma^{2}\right)\left(P_{21} G_{22}+\sigma^{2}\right) / G_{11} G_{12} .
\end{aligned}
$$

Since $A>0$ and independent of $P_{11}$, the solution of maximizing $J\left(P_{11}\right)$ is the solution for minimizing $-P_{11}^{2}+B P_{11}+C$. Note that $0 \leq P_{11} \leq P_{1, \max }$. If $B \leq 0,-P_{11}^{2}+B P_{11}+C$ is a monotonically decreasing function of $P_{11}$. Else if $B \geq$ $2 P_{1, \max },-P_{11}^{2}+B P_{11}+C$ is an increasing function of $P_{11}$. Otherwise, $-P_{11}^{2}+B P_{11}+C$ is a concave function of $P_{11}$. In either case, the maximum value of $J\left(P_{11}\right)$ is obtained at the boundary point of $P_{11}$, i.e., 0 or $P_{1, \max }$. Since $P_{11}+P_{12}=P_{1, \max }$, the optimal transmit power allocation vector $\mathbf{P}_{1}^{*}=\left[P_{11}^{*}, P_{12}^{*}\right]$ for cell 1 is $\left[P_{1, \max }, 0\right]$ or $\left[0, P_{1, \max }\right]$. Note that $\mathbf{P}_{1}^{*}$ is optimal for any given feasible power allocation vector $\mathbf{P}_{2}$. Hence, $\mathbf{P}_{1}^{*}$ is the optimal solution for (3), given $P_{11}+P_{12}=P_{1, \max }$. Thus, cell 1 transmits to only one user with its maximum transmit power. 
Based on Lemmas 1 and 2, we present the following theorem for the optimal power allocation solution.

Theorem 1. For the two-cell two-user CoMP joint transmission case, considering a random uniform phasing effect due to un-synchronized oscillators, the sum rate maximizing power allocation under per-cell power constraints is that each cell should transmit to only one user with its maximum transmit power. Mathematically, let $\triangle \mathcal{F}_{i}$ denote two sets of the corner points of the feasible domain for cell $i$, with $\triangle \mathcal{F}_{i}=\left\{\left[P_{i, \max }, 0\right],\left[0, P_{i, \max }\right]\right\}, i=1,2$. Then,

$$
\left[\mathbf{P}_{1}^{*}, \mathbf{P}_{2}^{*}\right]=\arg \max _{\mathbf{P}_{1} \in \triangle \mathcal{F}_{1}, \mathbf{P}_{2} \in \triangle \mathcal{F}_{2},} J\left(\mathbf{P}_{1}, \mathbf{P}_{2}\right) .
$$

Proof: Based on Lemma 1 and Lemma 2, at least one cell will transmit with full power to only one user. Without loss of generality, we assume that cell 1 transmits with full power to user 1, i.e., $\mathbf{P}_{1}^{*}=\left[P_{1, \max }, 0\right]$. Then, (3) becomes

$$
\begin{aligned}
\max _{\mathbf{P}_{2}} J\left(\mathbf{P}_{2}\right) & =\left(1+\frac{P_{1, \max } G_{11}+P_{21} G_{21}}{P_{22} G_{21}+\sigma^{2}}\right) \\
& \times\left(1+\frac{P_{22} G_{22}}{P_{1, \max } G_{12}+P_{21} G_{22}+\sigma^{2}}\right) \\
\text { s.t. } P_{21}+P_{22} & \leq P_{2, \max }, P_{21} \geq 0, P_{22} \geq 0 .
\end{aligned}
$$

In order to find $\mathbf{P}_{2}^{*}$, similar to the one in [3], by calculating the derivative of $J\left(\mathbf{P}_{2}\right)$ with respect to $P_{21}$, we have

$$
f\left(\mathbf{P}_{2}\right)=\frac{\partial J\left(\mathbf{P}_{2}\right)}{\partial P_{21}}=\frac{D\left(P_{21}+E\right)^{2}+F}{I},
$$

where

$$
\begin{aligned}
D & =G_{21} G_{22}^{2}>0, \\
E & =\left(P_{1, \max } G_{12}+\sigma^{2}\right) / G_{22}>0, \\
F & =P_{22} G_{22} G_{21}\left(P_{1, \max } G_{12}+\sigma^{2}\right) \\
& -P_{22} G_{22}^{2}\left(P_{1, \max } G_{11}+P_{22} G_{21}+\sigma^{2}\right), \\
I & =\left(P_{1, \max } G_{12}+P_{21} G_{22}+\sigma^{2}\right)^{2}\left(P_{22} G_{21}+\sigma^{2}\right)>0 .
\end{aligned}
$$

Since $I>0$, the solution of $f\left(\mathbf{P}_{2}\right)=0$ comes from the solution of $D\left(P_{21}+E\right)^{2}+F=0$. Note that $0 \leq P_{21} \leq P_{2, \text { max }}$. Hence, if $F \geq-D E^{2}, J\left(\mathbf{P}_{2}\right)$ is an increasing function of $P_{21}$. Else if $F \leq-D\left(P_{2, \max }+E\right)^{2}, J\left(\mathbf{P}_{2}\right)$ is a decreasing function of $P_{21}$. Otherwise, $D\left(P_{21}+E\right)^{2}+F$ has one zero for $P_{21}$ and changes from negative to positive when increasing $P_{21}$, i.e., there is a minimum point for $J\left(\mathbf{P}_{2}\right)$. In either case, the maximum value of $J\left(\mathbf{P}_{2}\right)$ is obtained at the boundary point of $P_{21}$. Using the same method, the above analysis also holds for $P_{22}$. Hence, $\mathbf{P}_{2}^{*}$ is found in the set of boundary points of the feasible domain, that is,

$$
\begin{aligned}
\mathbf{P}_{2}^{*} & \in\left\{P_{21}+P_{22}=P_{2, \max }, P_{21} \geq 0, P_{22} \geq 0\right\} \\
& \cup\left\{P_{21}=0,0 \leq P_{22} \leq P_{2, \max }\right\} \\
& \cup\left\{P_{22}=0,0 \leq P_{21} \leq P_{2, \max }\right\} .
\end{aligned}
$$

If $P_{21}^{*}=0$, the objective function in (7) becomes to maximize $J\left(P_{22}\right)=\left(1+\frac{P_{1, \max } G_{11}}{P_{22} G_{21}+\sigma^{2}}\right)\left(1+\frac{P_{22} G_{22}}{P_{1, \max } G_{12}+\sigma^{2}}\right)$ subject to $0 \leq P_{22} \leq P_{2, \max }$, where the maximum value is obtained when $P_{22}^{*}=P_{2, \max }$. Else if $P_{22}^{*}=0, J\left(\mathbf{P}_{2}\right)$ turns out to be a monotonically increasing function of $P_{21}$ in the domain of $0 \leq P_{21} \leq P_{2, \max }$, with the optimal solution achieved by $P_{21}^{*}=P_{2, \max }$. Otherwise, $P_{21}^{*}+P_{22}^{*}=$
$P_{2, \max }$ with $P_{21} \geq 0, P_{22} \geq 0$, and similar to Lemma 2, the solution of (7) is $\mathbf{P}_{2}^{*}=\left[P_{2, \max }, 0\right]$ or $\left[0, P_{2, \max }\right]$. In conclusion, assuming $\mathbf{P}_{1}^{*}=\left[P_{1, \max }, 0\right]$, the optimal solution of (3) is found by $\mathbf{P}_{2}^{*}=\left[P_{2, \max }, 0\right]$ or $\left[0, P_{2, \max }\right]$.

Due to the symmetry, we can conclude that the optimal solution for the considered worst case scenario is derived by (6). That is, depending on the noise and channel gains, each cell should transmit to only one user with its maximum transmit power. Note that joint transmission is still possible, when two cells select the same user for data transmission, e.g., $\mathbf{P}_{1}^{*}=\left[P_{1, \max }, 0\right]$ and $\mathbf{P}_{2}^{*}=\left[P_{2, \max }, 0\right]$.

\section{JOINT TRANSMISSION ANALYSIS}

According to (6), the optimal power allocation falls into one of the following four cases ${ }^{3}$ :

1) Two cells jointly transmit data to user 1 with full power;

2) Two cells jointly transmit data to user 2 with full power;

3) Cell $i$ transmits data to user $i$ with full power, $i=1,2$;

4) Cell $i$ transmits data to user $m$ with full power, $i \neq m$, $i=1,2$ and $m=1,2$.

In this section, we address the following questions: Under what kind of conditions will each case be the optimal solution? For what kind of system will joint transmission (case 1 and case 2) happen with high probability?

Assume that the two cells have the same maximum power constraint, $P_{\max }$. Let $R(n)$ denote the sum rate of case $n$, with $n=1,2,3,4$. Based on (2), we have

$$
\begin{aligned}
& R(1)=\log _{2}\left(1+\beta\left(G_{11}+G_{21}\right)\right), \\
& R(2)=\log _{2}\left(1+\beta\left(G_{12}+G_{22}\right)\right) \\
& R(3)=\log _{2}\left(\left(1+\frac{G_{11}}{G_{21}+1 / \beta}\right)\left(1+\frac{G_{22}}{G_{12}+1 / \beta}\right)\right), \\
& R(4)=\log _{2}\left(\left(1+\frac{G_{21}}{G_{11}+1 / \beta}\right)\left(1+\frac{G_{12}}{G_{22}+1 / \beta}\right)\right),
\end{aligned}
$$

where $\beta=P_{\max } / \sigma^{2}$. Let $\mathcal{G}(k)$ denote the feasible set of $R(k)=\max _{n} R(n)$, with $n=1,2,3,4$. Then, we have

$$
\begin{aligned}
& \mathcal{G}(1)=\left\{G_{x}>G_{y}, G_{x}>-c_{2}, G_{y}<c_{1}\right\} \\
& \mathcal{G}(2)=\left\{G_{x}<G_{y}, G_{x}<c_{1}, G_{y}>-c_{2}\right\} \\
& \mathcal{G}(3)=\left\{G_{x}>c_{1}, G_{y}>c_{1}\right\} \\
& \mathcal{G}(4)=\left\{G_{x}<-c_{2}, G_{y}<-c_{2}\right\}
\end{aligned}
$$

where $G_{x}=G_{11}-G_{12}, G_{y}=G_{22}-G_{21}, c_{1}=\beta G_{12} G_{21}>0$, $c_{2}=\beta G_{11} G_{22}>0$. Hence, the first question can be answered by (10), which is illustrated in Figure 2(a).

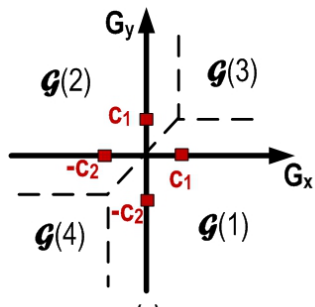

(a)

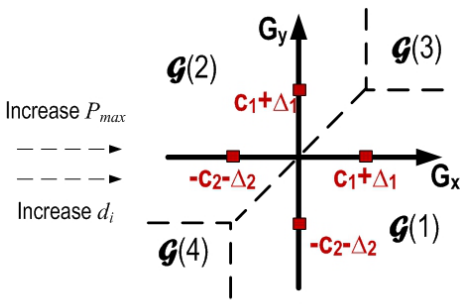

(b)
Figure 2. Feasible domain of the different optimal power allocation cases

${ }^{3}$ For cases 3 ) and 4), our system model turns out to be an interference channel, as each user can only be served by one cell. 
Note that $\mathcal{G}(1) \cup \mathcal{G}(2)$ is the joint transmission region, which increases with $c_{1}$ or $c_{2}$. Since $c_{1}$ and $c_{2}$ monotonically increase with $P_{\max }$, the joint transmission region increases with $P_{\max }$. On the other hand, assume that $P_{\max }$ is fixed. Since $c_{1}>0$, the channel power gains in region $\mathcal{G}(3)$ should satisfy $G_{11}>G_{12}$ and $G_{22}>G_{21}$, i.e., user $i$ is closer to the BS of cell $i, i=1,2$. Let $d_{i}$ denote the distance between user $i$ to its closer BS. When the two users move from the cell-center areas towards the overlapped cell-edge area, i.e., $d_{i}$ increases, it would be general that $G_{11}$ and $G_{22}$ decrease while $G_{12}$ and $G_{21}$ increase, leading to $G_{x}$ and $G_{y}$ decreasing while $c_{1}$ increasing. Thus, according to (10), the region of $\mathcal{G}(3)$ decreases when $d_{i}$ increases. For the region of $\mathcal{G}(4)$, the channel power gains should satisfy $G_{11}<G_{12}$ and $G_{22}<G_{21}$. In this case, the closer BS of user $i$ is cell $j$, with $i \neq j, i=1,2$ and $j=1,2$. Similarly, we can prove that the region of $\mathcal{G}(4)$ decreases when $d_{i}$ increases. Since $\mathcal{G}(1) \cup \mathcal{G}(2)$ is the complement set of $\mathcal{G}(3) \cup \mathcal{G}(4)$, the joint transmission region increases with $d_{i}$. Thus, we can conclude that the joint transmission region increases when the transmit power increases, or when the two users move from cell-center areas towards the overlapped cell-edge area (see Figure 2).

\section{Numerical Results}

The performance of the proposed power allocation scheme is studied by Monte-Carlo simulation, over 10,000 independent realizations of users' locations. Based on the system model described in Figure 1, we consider a two-cell two-user system with a radius of $R=500 \mathrm{~m}$ in each cell. The path loss is $L(d)=128.1+37.6 \log _{10}(d)$ in $\mathrm{dB}$. The shadowing standard deviation is $8 \mathrm{~dB}$, and the fast fading is Rayleigh distributed. The AWGN power is $-105 \mathrm{dBW}$. Assume that the two cells have the same maximum power constraint, $P_{\max }$.

Figure 3 (a) shows that the joint transmission probability increases with $P_{\max }$ when users are located in the overlapped cell-edge area. In Figure 3 (b), the joint transmission probability with different $d_{i}$ is plotted when $P_{\max }=20 \mathrm{~W}$. Two users are dropped along the dashed line in Figure 1 symmetrically. We can see that the joint transmission probability increases with $d_{i}$, in agreement with the conclusion made in section IV.

Figure 4 shows the sum rate with different $P_{\max }$ when users are located in the overlapped cell-edge area. Zero-forcing joint precoding (ZFJP) is shown as a benchmark assuming perfect synchronization [10]. Considering imperfect synchronization, the proposed power allocation scheme (proposed-PA) is compared with the equal power allocation (EPA), GPA [2], BPC [3], and Jo-WF [6]. The proposed-PA scheme achieves the best performance in the considered worst case scenario. Moreover, the crossing point in Figure 4 indicates that, when the transmit power is low, the Jo-WF scheme is superior to BPC by allowing joint transmission for one user. However, notice that interference is not taken into account in the JoWF scheme. Thus, when the transmit power is high (the interference becomes serious), BPC outperforms Jo-WF.

\section{CONCLUSIONS}

We address the downlink power allocation problem for a two-cell two-user joint transmission system, where syn-
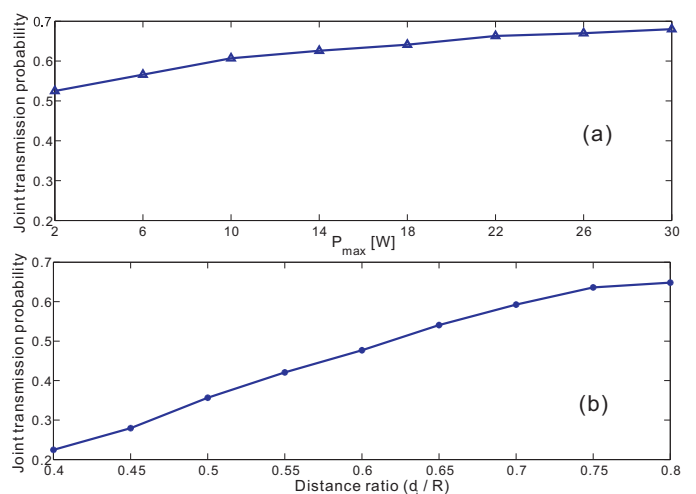

Figure 3. (a) Joint transmission probability vs. $P_{\max }$, (b) Joint transmission probability vs. distance ratio

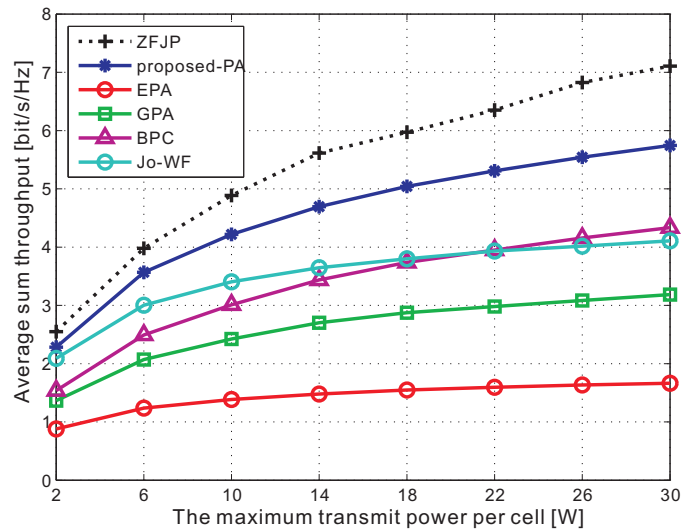

Figure 4. Average sum rate vs. maximum transmit power constraint per cell

chronization between base stations is extremely difficult. The derived solution has a simple feature, i.e., each cell transmits with full power to only one user. Numerical results show that the proposed scheme obtains significant gains over equal power allocation, binary power control, greedy power allocation and joint water-filling schemes. Extension to systems with more than two cells and two users is our ongoing work. Early results indicate that the proposed scheme is still optimal in the targeted scenario when applying a low SINR approximation.

\section{REFERENCES}

[1] A. J. Goldsmith, S.-G. Chua, "Variable-rate variable-power MQAM for fading channels," IEEE Trans. Commun., vol. 45, pp. 1218-1230, 1997.

[2] J. Jang and K. B. Lee, "Transmit power adaptation for multiuser OFDM systems", IEEE J. Select. Areas Commun., vol. 21, pp. 171-178, 2003.

[3] A. Gjendemsjø, D. Gesbert, G. Øien, S. Kiani, "Optimal power allocation and scheduling for two-cell capacity maximization," Proc. Modeling and Optimization in Mobile, Ad Hoc, and Wireless Networks, 2006.

[4] D. Gesbert, S. Hanly, H. Huang, S. Shamai Shitz, O. Simeone, and W. Yu, "Multi-cell MIMO cooperative networks: a new look at interference," IEEE J. Select. Areas Commun., vol. 28, pp. 1380-1408, 2010.

[5] H Huang, M Trivellato, A Hottinen, et al., "Increasing downlink cellular throughput with limited network MIMO coordination," IEEE Trans. Wireless Commun., vol. 8, pp. 2983-2989, 2009.

[6] B. Luo, Q. Cui, H. Wang, X. Tao, "Optimal joint water-filling for coordinated transmission over frequency-selective fading channels," IEEE Commun. Letters, vol. 15, no. 2, pp. 190-192, February 2011.

[7] J. Li, T. Svensson, C. Botella, et al., "Joint scheduling and power control in coordinated multi-point clusters," Proc. IEEE VTC'11, 2011.

[8] V. Jungnickel, T. Wirth, M. Schellmann, et al., "Synchronization of cooperative base stations," Proc. IEEE ISWCS'08, 2008.

[9] R. Krishnan, M. R. Khanzadi, L. Svensson, T. Eriksson, and T. Svensson, "Variational bayesian framework for receiver design in the presence of phase noise in MIMO systems," Proc. IEEE WCNC, 2012.

[10] F. Boccardi and H. Huang, "Zero-forcing precoding for the MIMO broadcast channel under per-antenna power constraints," Proc. IEEE SPAWC'06, 2006. 